\title{
Numerical solution of the Duffing equation with random coefficients
}

\author{
Marcin Kamiński • Alberto Corigliano
}

Received: 20 March 2014/ Accepted: 18 February 2015/Published online: 28 February 2015

(C) The Author(s) 2015. This article is published with open access at Springerlink.com

\begin{abstract}
The main aim of this work is numerical solution of the nonlinear vibrations of micro-resonators exhibiting bounded and Gaussian uncertainty in their parameters. The mechanical response in deterministic situation is described by the Duffing equation, whose numerical solution is obtained with the Runge-Kutta-Fehlenberg algorithm, while probabilistic analysis is carried out using the generalized stochastic perturbation technique enriched with automatic optimization of the approximating polynomial. Basic solution to this nonlinear vibration in the deterministic context is obtained with the use of the computer algebra system MAPLE, where all additional probabilistic procedures are also implemented. We compare each time expectations, coefficients of variation, skewness and kurtosis for the structural response to show probabilistic sensitivity of the MEMS accelerometer with respect to its design parameter expectation and coefficient of variation. An additional comparison of the proposed technique
\end{abstract}

\section{Kamiński ( $\square)$}

Chair of Structural Reliability, Department of Structural Mechanics, Technical University of Łódź, Al.

Politechniki 6, 90-924 Łódź, Poland

e-mail: Marcin.Kaminski@p.lodz.pl

\section{A. Corigliano}

Department of Civil and Environmental Engineering, Politecnico di Milano, Piazza Leonardo da Vinci 32, 20133 Milan, Italy

e-mail: alberto.corigliano@polimi.it with the traditional Monte-Carlo sampling for the first four probabilistic moments is also provided.

Keywords MEMS $\cdot$ Microsystems $\cdot$ Nonlinear vibration · Duffing equation · Microresonators · Stochastic perturbation technique $\cdot$ Symbolic computing

\section{Introduction}

Micro-electro-mechanical systems (MEMS) [5] are crucial nowadays for micro-gyroscopes and accelerometers [22], mobile communication [21], building and designing of new computers [25], precise detection of the vibrations and fatigue [8] (structural inspection and monitoring), also in superconductors [24] as well as in various optics practical problems solutions, like image stabilization in digital photography [15]. A special role in this area belongs to vibrating micro-beams that are subjected to the coupled micro-electro-mechanical fluctuating field, so that a precise mathematical model for their electromechanical behavior is crucial for complete understanding and optimal design of such devices. Usually, a single I-beam is modeled mathematically (numerically) using the vibrating single degree of freedom system, governed by the Duffing equation [12, 14], where non-linear effects have multi-field character (especially damping) [9, 13]. Solutions for the 
Duffing equation exist for some specific combinations of the parameters and forcing functions. A variety of computational experiments with symbolic computing programs show that this is a very challenging task, even in the deterministic context, while only few reliable analytical solutions are available [7]. We use for this purpose the Runge-Kutta-Fehlenberg algorithm implemented also in the library of differential equations in the program MAPLE.

The problem complicates significantly once some uncertainty is taken into account in the mathematical model, where especially geometrical imperfections of the devices, material parameters variations [1] (connected also with coupled field phenomena), temperature variations as well as shock, impact and fatigue [3, 8] may have a decisive role for their reliability and durability [2,6]. The problems of structural dynamics under stochastic excitation [12, 17] and/or the vibrations with some design parameters treated as random variables have been solved many times before by a variety of methods including a number of theoretical derivations [19], KarhunenLoeve expansions [4], fuzzy sets theory [16] and also lower order perturbation method. There are also several computational strategies available in this area [18] like widely known Monte-Carlo simulation technique (in crude, stratified, Metropolis or some twister versions) as well as the worst scenario strategy, polynomial chaos driven Stochastic Finite Elements [1] or the recently developed Approximated Deformation Principal Modes (APDM) approach [20]. It is known however that the simulation strategy is extremely time consuming and has some minor points considering statistical estimation procedures, while the remaining techniques do not allow for higher probabilistic moments and coefficients determination. That is why we propose to make use of a new technique that overcomes these problems, called the generalized stochastic perturbation technique [10]. It is based on general order Taylor expansion of all random input parameters and output state functions, several solutions to the initial deterministic problem with varying value of the given uncertainty source (like for polynomial chaos technique) and numerical recovery of the response function relating output-toinput parameters being some higher order polynomial of the random input. The well known formulation is enriched in this paper with a determination of an optimal polynomial degree that minimizes both the
RMS error and correlation coefficient inherent in the least squares approximation; finally, we computationally determine up to the first four probabilistic moments and coefficients of the desired structural response. This strategy is tested on two different cases - the first one concerns damped vibrations of a linear oscillator and it serves rather for a comparison with the Monte-Carlo simulation scheme, the second one concerns a case study devoted to the forced vibration of a micro-beam exhibiting stochastic damping. An application of this strategy to coupled field Finite Element Method analysis has been provided before in [11]. It should be mentioned that an application of the perturbation technique itself in the deterministic context is well known from the solution of various problems in dynamics. The method proposed here contains very similar apparatus, where the perturbations are considered with respect to the expected value of the structural response unlike in deterministic situation, where they were analyzed in addition to the equilibrium state.

We present in this paper numerical analysis of the Duffing equation with random parameter showing a form convenient to the perturbation-based symbolic analysis together with the perturbation-based definitions of basic probabilistic characteristics computed. We apply this approach to L-shaped micro-resonator discussed in [22] with random damping to investigate its first four probabilistic characteristics of the displacements and velocities. We define the damping coefficient as the Gaussian random variable, however our analysis is valid for the truncated Gaussian variable (with negligible error), symmetric distributions (non-Gaussian distributions need more than the first two probabilistic moments but expansion remains the same) and even non-symmetric variables (like lognormal, where full expansions are necessary). Computational determination of the first four probabilistic moments is preferred as we can verify the output uncertainty level versus the input one as well as check if the output distribution may be treated as Gaussian also, which significantly simplifies further analysis. An original aspect of this work is in the application of the higher order stochastic perturbation technique that allows for determination of up to the fourth order probabilistic characteristics of the dynamic response (especially higher order statistics like skewness and kurtosis). The Response Function Method is statistically optimized in the sense that the 
order of approximating polynomial minimizes standard error and at the same time maximizes the correlation of this response to the given set of discrete solutions of the original Duffing equation. A comparison of such a methodology against the Monte-Carlo simulation gives unique opportunity to initially confirm an applicability of such a higher order optimized stochastic perturbation technique in highly nonlinear transient problems.

\section{Governing equations}

We consider forced nonlinear vibrations of a single d.o.f. mechanical system governed by the Duffing equation in the following form [12]:

$$
\begin{aligned}
& m \ddot{x}(t)+c \dot{x}(t)+k_{1} x(t)+k_{2} x^{2}(t)+k_{3} x^{3}(t) \\
& \quad=F \sin (\omega t) .
\end{aligned}
$$

In Eq. (1) $x$ is the displacement, the upper dots are equivalent to time derivative(s), $m$ denotes the mass of the vibrating structure, $c$ stands for the damping coefficient, $k_{1}, k_{2}$ and $k_{3}$ are first, second, and third order stiffness coefficients related to various physical fields and sources, $F$ and $\omega$ are the amplitude and frequency of the forcing signal. It is well-known that the general solution to this differential equation depends strongly upon a combination of its coefficients and may return essentially different phase portraits. As we are interested in a computational recovery of the semi-analytical dynamic response function in-between the triplets $\ddot{x}(t), \dot{x}(t), \quad x(t)$ and the structural design parameters like $m, c, k_{1}, k_{2}$ and $k_{3}$, we solve this equation iteratively for various combinations of these parameters, indexing this equation with $i=1, \ldots, N$ :

$$
\begin{aligned}
& m_{(i)} \ddot{x}_{(i)}(t)+c_{(i)} \dot{x}_{(i)}(t)+k_{1(i)} x_{(i)}(t) \\
& \quad+k_{2(i)} x_{(i)}^{2}(t)+k_{3(i)} x_{(i)}^{3}(t)=F \sin (\omega t) .
\end{aligned}
$$

We use traditional definitions to compute basic probabilistic moments and characteristics for the structural response at $\tau \in[0, \infty)$ and for a given input random parameter $b$ with its probability density $p_{b}(y)$; these are [10, 23]:

expected values

$$
E[x(b ; \tau)]=\int_{-\infty}^{+\infty} x(b ; \tau) p_{b}(y) d y
$$

$r$ th central probabilistic moments

$\mu_{r}(x(b ; \tau))=\int_{-\infty}^{+\infty}(x(b ; \tau)-E[x(b ; \tau)])^{r} p_{b}(y) d y$.

Additionally, we introduce the coefficients of variation, $\alpha$, skewness $\beta$ and kurtosis $\kappa$ as

$$
\begin{aligned}
\alpha(x(b ; \tau)) & =\sqrt{\frac{\operatorname{Var}(x(b ; \tau))}{(E[x(b ; \tau)])^{2}}} \\
\beta(x(b ; \tau)) & =\frac{\mu_{3}(x(b ; \tau))}{(\sqrt{\operatorname{Var}(x(b ; \tau))})^{3}}, \\
\kappa(x(b ; \tau)) & =\frac{\mu_{4}(x(b ; \tau))}{(\operatorname{Var}(x(b ; \tau)))^{2}}-3 .
\end{aligned}
$$

The corresponding definitions and formulas are applicable to the first four probabilistic moments of $\ddot{x}(t), \quad \dot{x}(t)$. We use the generalized stochastic perturbation technique [10] based on the $n$th order probabilistic expansion of all variables and time response via Taylor series about their mean values, so that the time response of the system at the specific time $\tilde{\tau} \in[0, \infty)$ is expanded for instance as (with traditionally adopted $\varepsilon=1$ )

$x(b ; \tilde{\tau})=x^{0}\left(b^{0} ; \tilde{\tau}\right)+\sum_{j=1}^{n} \frac{\varepsilon^{j}}{j !} \Delta b^{j} D_{b}^{j}(x(\tilde{\tau}))$,

where $D_{b}^{j}(x(\tilde{\tau}))$ serves for partial derivative of the dynamic response $x(\tilde{\tau})$ of the $j$ th order with respect to the random parameter $b$ (to shorten significantly all the perturbation-based formulas). It is important to notice that partial derivatives of the structural dynamic response with respect to the given input random parameter are calculated at its mean value in the traditional deterministic manner. Since an analytical interrelation of this dynamic response with respect to the chosen input random parameter is usually implicit, we apply the Weighted Least Squares Method (WLSM) [10] here, to approximate this function numerically [11]. Inserting this expansion into the definitions (3-4) brings for the Gaussian distributions the following results for the expectations and variances of the same function: 


$$
\begin{aligned}
E[x(b ; \tau)]= & \int_{-\infty}^{+\infty} x(b ; \tau) p_{b}(y) d y=x^{0}\left(b^{0} ; \tau\right) \\
& +\frac{\varepsilon^{2}}{2} D_{b}^{2}(x(\tau)) \mu_{2}(b) \\
& +\frac{\varepsilon^{4}}{4 !} D_{b}^{4}(x(\tau)) \mu_{4}(b)+\frac{\varepsilon^{6}}{6 !} D_{b}^{6}(x(\tau)) \mu_{6}(b) \\
& +\frac{\varepsilon^{8}}{8 !} D_{b}^{8}(x(\tau)) \mu_{8}(b)+\frac{\varepsilon^{10}}{10 !} D_{b}^{10}(x(\tau)) \mu_{10}(b) .
\end{aligned}
$$

Consecutively, we apply the definition of the variance

$$
\begin{aligned}
\operatorname{Var}(x(b ; \tau)) & =\mu_{2}(x(b ; \tau)) \\
& =\int_{-\infty}^{+\infty}(x(b ; \tau)-E[x(b ; \tau)])^{2} p_{b}(y) d y
\end{aligned}
$$

to derive its perturbation-based formula including higher order derivatives of the response function. Simplifying the notation $D_{b}^{n} \equiv D_{b}^{n}(x(b ; \tau))$ and inserting $\varepsilon=1$ returns:

$$
\begin{aligned}
\operatorname{Var}(x(b ; \tau))= & \mu_{2}(b)\left(D_{b}\right)^{2}+\mu_{4}(b)\left\{\frac{1}{4}\left(D_{b}^{2}\right)^{2}+\frac{1}{3} D_{b}^{3} D_{b}^{1}\right\} \\
& +\mu_{6}(b)\left\{\frac{1}{36}\left(D_{b}^{3}\right)^{2}+\frac{1}{24} D_{b}^{4} D_{b}^{2}+\frac{1}{60} D_{b}^{5} D_{b}^{1}\right\} \\
& +\mu_{8}(b)\left\{\frac{1}{576}\left(D_{b}^{4}\right)^{2}+\frac{1}{360} D_{b}^{5} D_{b}^{3}\right. \\
& \left.+\frac{1}{2520} D_{b}^{7} D_{b}^{1}+\frac{1}{720} D_{b}^{6} D_{b}^{2}\right\} \\
& +\mu_{10}(b)\left\{\frac{1}{14400}\left(D_{b}^{5}\right)^{2}+\frac{1}{40320} D_{b}^{8} D_{b}^{2}\right. \\
& \left.+\frac{1}{8640} D_{b}^{6} D_{b}^{4}+\frac{1}{15120} D_{b}^{7} D_{b}^{3}+\frac{1}{181440} D_{b}^{9} D_{b}^{1}\right\} .
\end{aligned}
$$

Determination of higher central probabilistic moments and related coefficients proceeds in a similar way and can be implemented in symbolic computing software using "taylorization" procedure inherent for numerous computer algebra systems [10] including MAPLE. One may obtain for the third and fourth central probabilistic moments:

$$
\begin{aligned}
\mu_{3}(x(b ; \tau))=\int_{-\infty}^{+\infty}(x(b ; \tau)-E[x(b ; \tau)])^{3} p_{b}(y) d y \\
=\frac{3}{2} \mu_{4}(b)\left(D_{b}\right)^{2} D_{b}^{2} \\
+\mu_{6}(b)\left\{\frac{1}{8}\left(D_{b}\right)^{3}+\frac{1}{2} D_{b} D_{b}^{2} D_{b}^{3}+\frac{1}{8}\left(D_{b}\right)^{2} D_{b}^{4}\right\} \\
+\mu_{8}(b)\left\{\frac{1}{24} D_{b} D_{b}^{3} D_{b}^{4}+\frac{1}{40} D_{b} D_{b}^{2} D_{b}^{5}\right. \\
\left.+\frac{1}{240}\left(D_{b}\right)^{2} D_{b}^{6}+\frac{1}{32}\left(D_{b}^{2}\right)^{2} D_{b}^{4}+\frac{1}{24}\left(D_{b}^{3}\right)^{2} D_{b}^{2}\right\} \\
+\mu_{10}(b)\left\{\frac{1}{480} D_{b} D_{b}^{4} D_{b}^{5}+\frac{1}{1680} D_{b} D_{b}^{2} D_{b}^{7}\right. \\
\left.+\frac{1}{720} D_{b} D_{b}^{3} D_{b}^{6}\right\} \\
+\mu_{10}(b)\left\{\frac{1}{240} D_{b}^{2} D_{b}^{3} D_{b}^{5}+\frac{1}{13440}\left(D_{b}\right)^{2} D_{b}^{8}\right. \\
\left.+\frac{1}{960}\left(D_{b}^{2}\right)^{2} D_{b}^{6}+\frac{1}{384}\left(D_{b}^{4}\right)^{2} D_{b}^{2}+\frac{1}{288}\left(D_{b}^{3}\right)^{2} D_{b}^{4}\right\}
\end{aligned}
$$

and

$$
\begin{aligned}
\mu_{4}(x(b ; \tau))=\int_{-\infty}^{+\infty}(x(b ; \tau)-E[x(b ; \tau)])^{4} p_{b}(y) d y \\
=\mu_{4}(b)\left(D_{b}\right)^{4}+\mu_{6}(b)\left\{\frac{3}{2}\left(D_{b}\right)^{2}\left(D_{b}^{2}\right)^{2}+\frac{2}{3}\left(D_{b}\right)^{3} D_{b}^{3}\right\} \\
+\mu_{8}(b)\left\{\frac{1}{16}\left(D_{b}^{2}\right)^{4}+\frac{1}{30}\left(D_{b}\right)^{3} D_{b}^{5}+\frac{1}{6}\left(D_{b}\right)^{2}\left(D_{b}^{3}\right)^{2}\right. \\
\left.+\frac{1}{4}\left(D_{b}\right)^{2} D_{b}^{2} D_{b}^{4}+\frac{1}{2} D_{b}\left(D_{b}^{2}\right)^{2} D_{b}^{3}\right\} \\
+\mu_{10}(b)\left\{\frac{1}{1260}\left(D_{b}\right)^{3} D_{b}^{7}+\frac{1}{96}\left(D_{b}\right)^{2}\left(D_{b}^{4}\right)^{2}\right. \\
\left.+\frac{1}{54} D_{b}\left(D_{b}^{3}\right)^{3}+\frac{1}{48} D_{b}^{4}\left(D_{b}^{2}\right)^{3}+\frac{1}{24}\left(D_{b}^{2}\right)^{2}\left(D_{b}^{3}\right)^{2}\right\} \\
+\mu_{10}(b)\left\{\frac{1}{12} D_{b} D_{b}^{2} D_{b}^{3} D_{b}^{4}+\frac{1}{60}\left(D_{b}\right)^{2} D_{b}^{3} D_{b}^{5}\right. \\
\left.+\frac{1}{120}\left(D_{b}\right)^{2} D_{b}^{2} D_{b}^{6}+\frac{1}{40} D_{b}\left(D_{b}^{2}\right)^{2} D_{b}^{5}\right\}
\end{aligned}
$$

This technique may also serve for the non-Gaussian responses and then one needs to complete these 
expansions with the odd order terms, which extends almost twice the formulas inserted above. Finally, it is necessary to point out that the polynomial response functions are determined separately in each discrete time instant, so that their coefficients are time dependent, while further numerical analysis may include the case, where the degree of approximating random polynomial also vary in time. Therefore, the overall computer effort and time consumption in the stochastic approach proposed is mainly affected by the time increment chosen in the Runge-Kutta-Fehlenberg solution to the nonlinear vibrations.

\section{Computational analysis}

\subsection{Perturbation method validation test}

The linear oscillator initially subjected to the perturbation-based randomization procedure is proposed as

$m \frac{d^{2} x_{1}(t)}{d t^{2}}+c \frac{d x_{1}(t)}{d t}+k x_{1}(t)=Q \sin (t)$

where $\mathrm{m}=0.3965, \mathrm{k}=0.761$ and $\mathrm{Q}=2.52 \mathrm{E}-9$. The damping coefficient is treated here as the Gaussian random variable having the expected value equal to $E[c]=0.00389$ and coefficient of variation equal to $\alpha(\mathrm{c})=0.10$ [defined in formula (5)] to provide a comparison of the tenth order stochastic perturbation technique proposed with the classical Monte-Carlo scheme. An uncertainty in viscous part is considered here according to a number of important technical applications of such a model; not necessarily Gaussian of course. Computer analysis is performed here entirely in the package MAPLE with the use of 11 computing cycles about the expected value and weighting scheme adjacent to an importance distribution $[1,6]$; they are equivalent to the following series of damping coefficients $c_{i}=0.00394 \pm n 0.00002$, where $\mathrm{n}=1, \ldots, 4$. The resulting vibrations (left graph) and phase portrait (right graph) obtained for the mean value of the coefficient $c$ are given below in Fig. 1 for the first $50 \mathrm{~s}$ of the forced vibrations process, where numerical solution is found with the time step $\Delta \mathrm{t}=1 \mathrm{~s}$. The fourth order approximating polynomial for the displacements response functions has been adopted according to the WLSM optimization procedure attached to the next experiment. The overall computational effort in the perturbation-based experiment is equivalent to $80.36 \mathrm{MB}$ and $7.34 \mathrm{~s}$ for the entire stochastic perturbation based solution discussed below (with ten numbers precision). The crude MonteCarlo simulation scheme for the contrast is based on $2 \times 10^{5}$ samples and costs $2093.34 \mathrm{~s}$ with 527.65 MBs carried out with eight numbers precision (provided according to memory limitations).

First of all we compare the expectations of displacements computed according to the perturbation method (left graph of Fig. 2) with the corresponding mean values estimated via the MonteCarlo simulation (the right graph of Fig. 2). As one may notice, both extreme values, their timings as well as the patterns of both dynamic responses are extremely similar to each other. Further modifications of the input coefficient of variation (both increasing and decreasing) omitted here for the
Fig. 1 Linear oscillator vibrations and their phase portrait
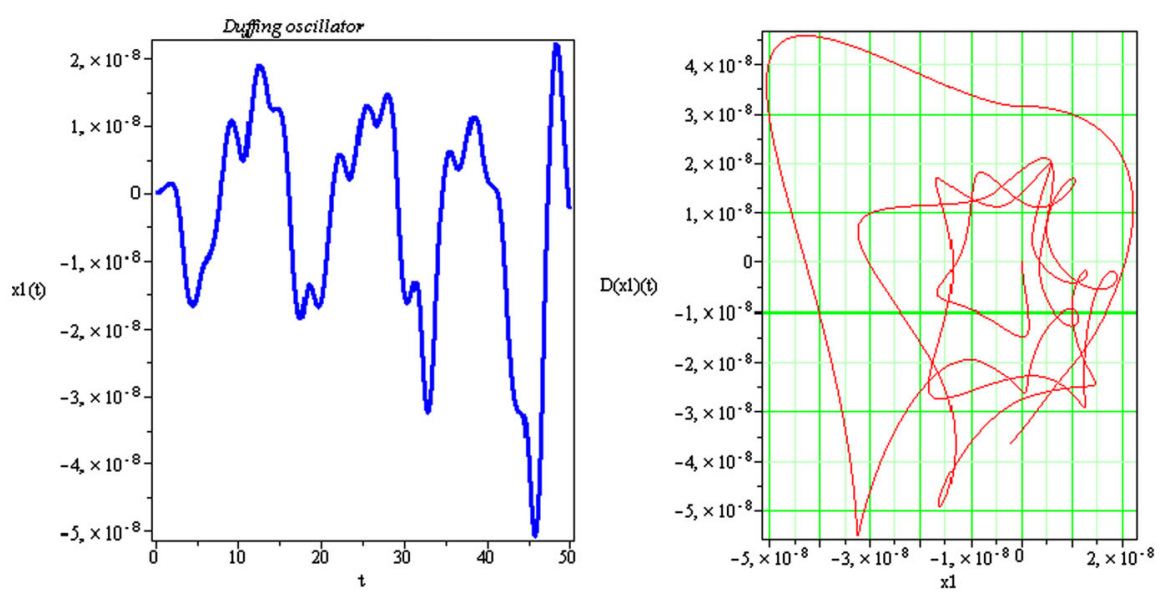
Fig. 2 Expected values of the dynamic response via the stochastic perturbation technique (left) and the Monte-Carlo simulation scheme (right)
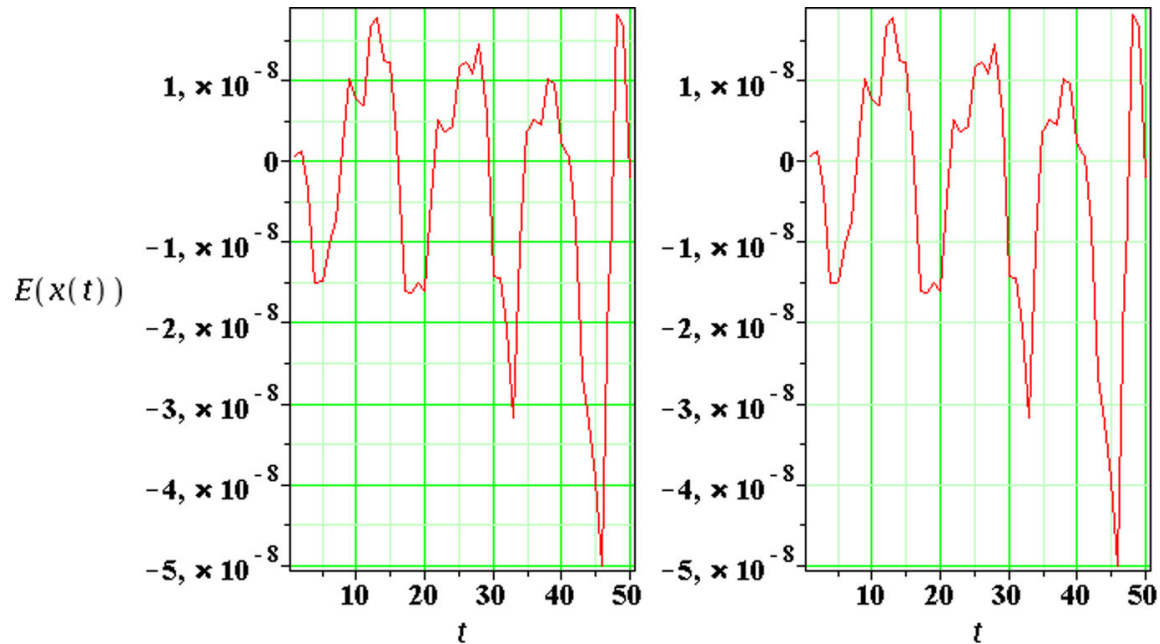

brevity of a presentation do not affect this similarity. Furthermore, time variations of the coefficients of variation, skewness and kurtosis of the displacements are attached in Figs. 3, 4 and 5 determined by using of the stochastic tenth order perturbation scheme (left series) and, independently, via the Monte-Carlo simulation scheme (right series). The most apparent difference to the well documented previous models available in linear elasticity [11] is an enormous increase of the resulting extreme coefficient of variation for displacements (Fig. 3) which is close to 1.5 and this means 15 times larger than the input value of this parameter. It is dramatic uncertainty of these displacements in a very specific moment of these vibrations and we notice that this is some local extreme by only, while the rest of the vibrations is accompanied by $\alpha(x(t))$ close to 0.20 rather. The second order characteristics determined with the use of the perturbation method and MonteCarlo scheme coincide perfectly with each otherboth extreme values as well as the pattern and particular time fluctuations are the same. A comparison of the skewness (Fig. 4) and kurtosis (Fig. 5) is not so perfect, because although the patterns returned by stochastic perturbation and, independently, simulation methods are very similar to each other, the extreme values are different. It looks that the stochastic perturbation technique underestimates these extremes, but this happens only once or twice in the given period of time; the remaining magnitudes coincide with each other. Analyzing this comparison one needs to recall the fact that the Monte-Carlo simulation exhibits statistical convergence of the probabilistic moments and coefficients to their real values and a weaker comparison in case of higher order statistics may result from computational discrepancies in both techniques at the same time.

\subsection{Stochastic MEMS modeling}

The vibrating system under study is shown in Fig. 6; it is represented by a so-called L-shaped resonator and was discussed in [22] where its response was compared to experimental results, after obtaining an equivalent 1 d.o.f. dynamic model. We solve here the same boundary-initial problem in the probabilistic context, where the mass, the first, second and third order equivalent stiffnesses are considered here as the input design parameters. Taking into account the mechanical and electrical contributions $k_{\mathrm{m}}$ and $k_{\mathrm{e}}$, the stiffness coefficients $k_{i}, i=1,2,3$ are computed as [22]:

$$
\begin{aligned}
k_{1} & =\left(k_{\mathrm{m} 1}-k_{\mathrm{e} 1}\right)=(0.829-0.068) \mathrm{N} / \mathrm{m}=0.761 \mathrm{~N} / \mathrm{m}, \\
k_{2} & =0 \mathrm{~N} / \mathrm{m}^{2} \\
k_{3} & =\left(k_{\mathrm{m} 3 \mathrm{~L}}-k_{\mathrm{e} 3}\right)=\left(1.45 \times 10^{11}-2.2 \times 10^{10}\right) \mathrm{N} / \mathrm{m}^{3} \\
& =12.3 \times 10^{10} \mathrm{~N} / \mathrm{m}^{3}
\end{aligned}
$$

The effective mass of the micro-resonator was calculated in [22] from its length $L=400 \mu \mathrm{m}$, width $t=1.2 \mu \mathrm{m}$, out of the plane thickness $w=15 \mu \mathrm{m}$ and the silicon mass density $\rho=2330 \frac{\mathrm{kg}}{\mathrm{m}^{3}}$. The value $m=0.3965 \times M=6.65 \times 10^{-12} \mathrm{~kg}$ is obtained, 
Fig. 3 Coefficients of variation of the dynamic response via the stochastic perturbation technique (left) and the Monte-Carlo simulation scheme (right)

Fig. 4 Skewness of the dynamic response via the stochastic perturbation technique (left) and the Monte-Carlo simulation scheme (right)

Fig. 5 Kurtosis of the dynamic response via the stochastic perturbation technique (left) and the Monte-Carlo simulation scheme (right)
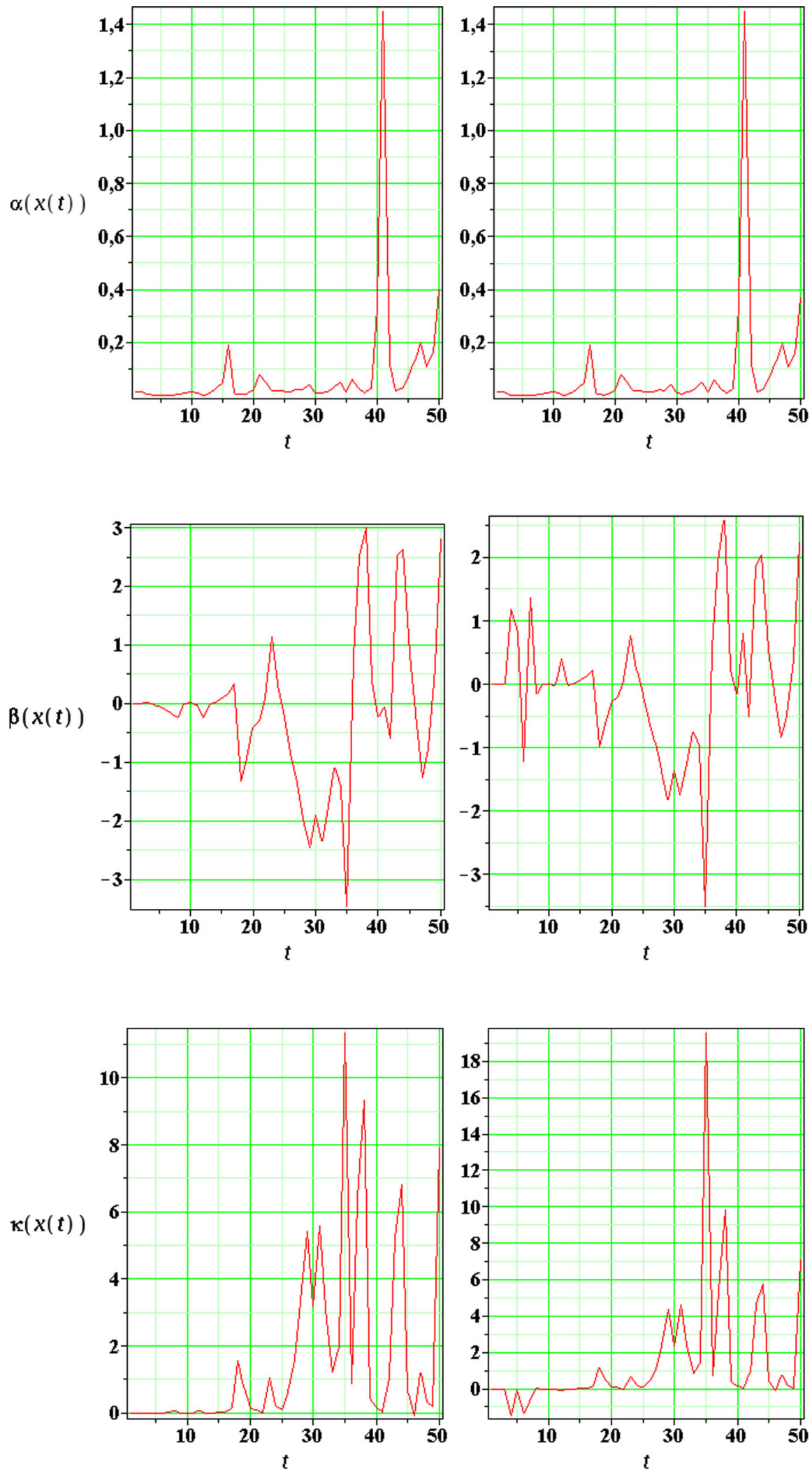
Fig. 6 Micro-resonator subjected to stochastic excitation [16]

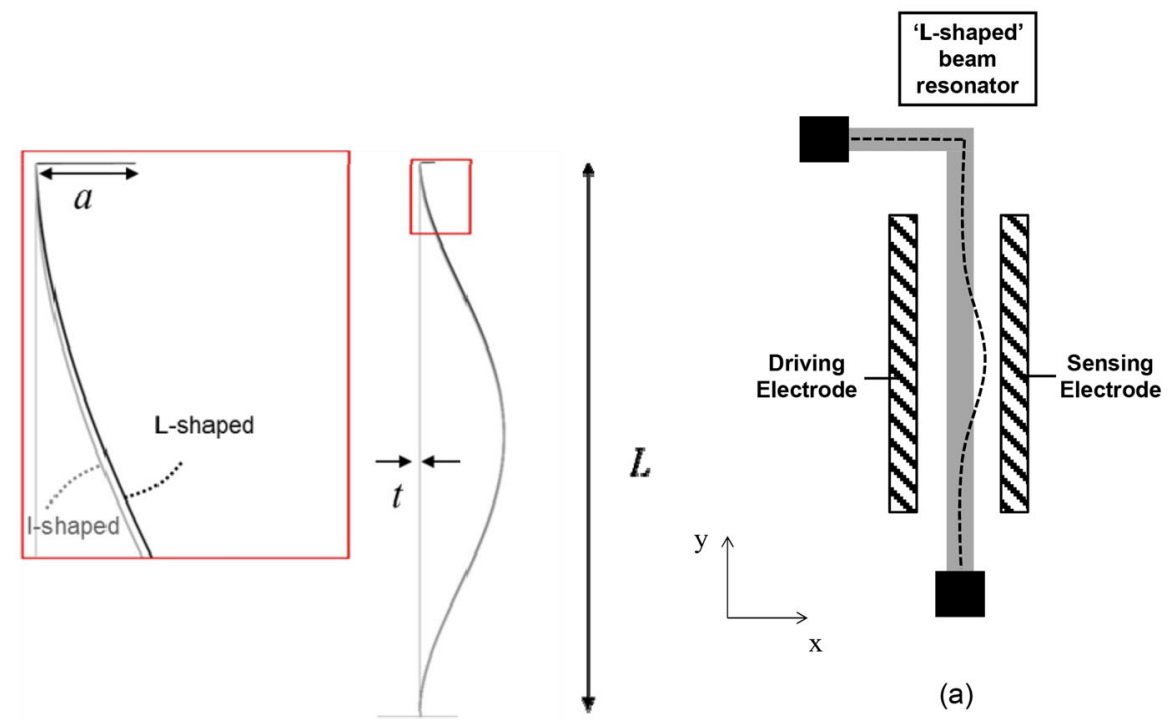

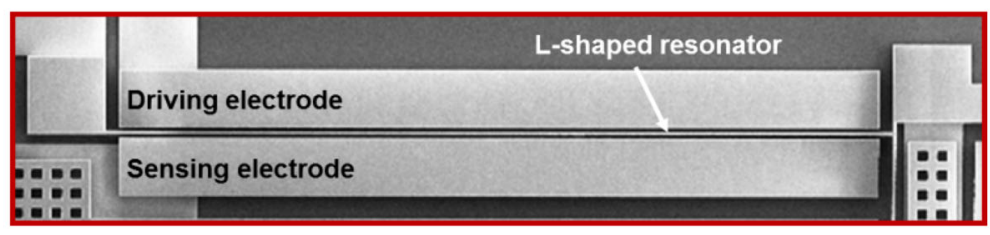

using the formula that gives the equivalent mass $m$ as a fraction of the total beam mass $M=16.78 \times$ $10^{-12} \mathrm{~kg}$. The damping coefficient is the random input parameter; its mean value has been initially evaluated from the formula $c=\frac{1}{Q} \sqrt{\mathrm{km}} \quad\left[\frac{\mathrm{Nsec}}{\mathrm{m}}\right]$, where $k$ includes all the stiffnesses introduced in Eq. (13) and then by selecting four possible values of the quality factor $Q=[100,210,1000,10000]$, where the value of 210 is the quality factor measured for the device discussed in [22]. Therefore, the expected values of the damping parameter $c$ are taken as equal to $E[c]=[0.0235,0.0112,0.00235,0.000235] \times 10^{-6}$ $[\mathrm{Nsec} / \mathrm{m}]$ and the coefficient of variation of this physical parameter is taken further from the interval $\alpha(c) \in[0.00,0.20]$ [10]. The external forcing function is assumed to have the harmonic form $F \sin (\omega t)$ and we adopt natural initial conditions as $x(t=0)=$ $0, \frac{d x(t=0)}{d t}=0$. An external force due to the electrostatic actuation is considered in the following form (see [16]):

$F \sin (\omega t)=\eta v_{a}(t)$ where

$$
\begin{aligned}
& \eta=\bar{\alpha} V_{p} \frac{\varepsilon_{0} w L}{d^{2}}, \quad \bar{\alpha}=0.523, \quad V_{p} \\
& =2 \div 9[V], \quad \varepsilon_{0} \\
& =8.8541878176 \times 10^{-12}[\mathrm{~F} / \mathrm{m}] \text {. } \\
& w=15 \mu m, L=400 \mu m, d=2.1 \mu m, \\
& v_{a}(t)=v_{a} \sin (\omega t), \quad v_{a}=5 \times 10^{-3} \div 1 \times 10^{-1}[V] \text {. }
\end{aligned}
$$

In the above relations $\bar{\alpha}$ denotes the coefficient related to the mechanical behavior of the resonator, $V_{p}$ is the bias voltage, $\varepsilon_{0}$ is the absolute vacuum permittivity constant, $d$ is the gap between the oscillating beam and the electrode, $v_{a}(t)$ is the actuation voltage, usually modulated at the mechanical frequency of the oscillating beam $\omega$. Finally, the external force has the following multiplier: $F=56.7 \times 10^{-10}[N]$, while $\omega$ is adopted as $10^{3}$. The entire computational analysis in both deterministic and probabilistic context has been provided in the computer algebra package MAPLE, v. 14. Firstly, four 
different deterministic spectra obtained with the Runge-Kutta-Fehlenberg algorithm for corresponding expectations of the damping coefficient as listed above and given in Figs. 7 and 8.

As it is documented in Figs. 7 and 8 (and also consistent with engineering intuition), the larger the damping coefficient, the smaller the amplitude of this vibration spectrum. The largest damping coefficient results in almost perfectly periodic displacements with time independent amplitude, while the hundred times smaller (right diagram in Fig. 8) results in non-periodic motion with an amplitude increasing moderately in time. Let us note that this amplitude in a very short initial time of the MEMS vibration increases almost three times. Then, we compute the expectations, coefficients of variations, skewness and kurtosis histories for both micrometer displacements and velocities-they are given in Figs. 9, 10, 11 and 12; they are all computed consequently using the tenth order stochastic perturbation technique described in the previous section. They are determined after 11 various deterministic solutions of the original Eq. (1) with damping coefficient varying uniformly within the few percents large neighborhood of its expectation and it is repeated four times for different input expectations of this parameter. This method is based upon the LSM procedure implemented in the system MAPLE, where the optimal degree of the polynomial response function is chosen by a minimization of the correlation and RMS error in this approximation. These parameters are contrasted in Table 1 for various orders of the least squares approximants (from the first up to the tenth) and this comparison justifies precisely a choice of the fourth order of the dynamic response functions relating the displacements at the given time to the damping coefficient $c$.

First of all it is seen in Fig. 9 that expected values of the resulting excitation show different sensitivity with
Fig. 7 Displacements [m] for $c=0.0235 \times 10^{-6}$ $[\mathrm{Nsec} / \mathrm{m}]($ left $)$ and $0.0112 \times 10^{-6}$ (right)
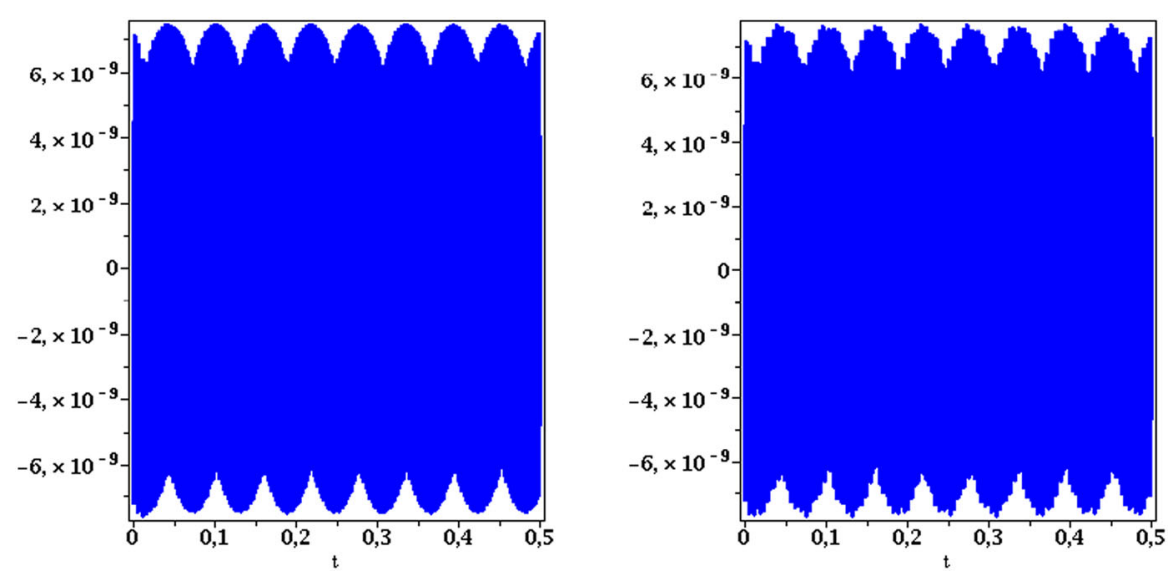

Fig. 8 Displacements [m] for

$c=0.00235 \times 10^{-6}[\mathrm{Nsec} /$ $\mathrm{m}]$ (left) and $0.000235 \times 10^{-6}($ right $)$

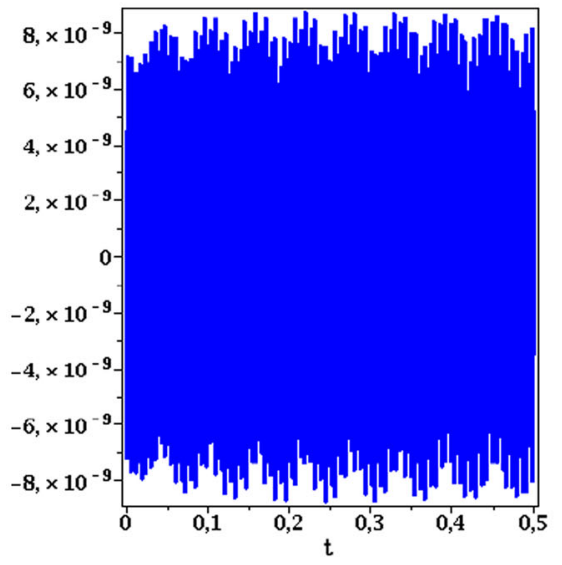




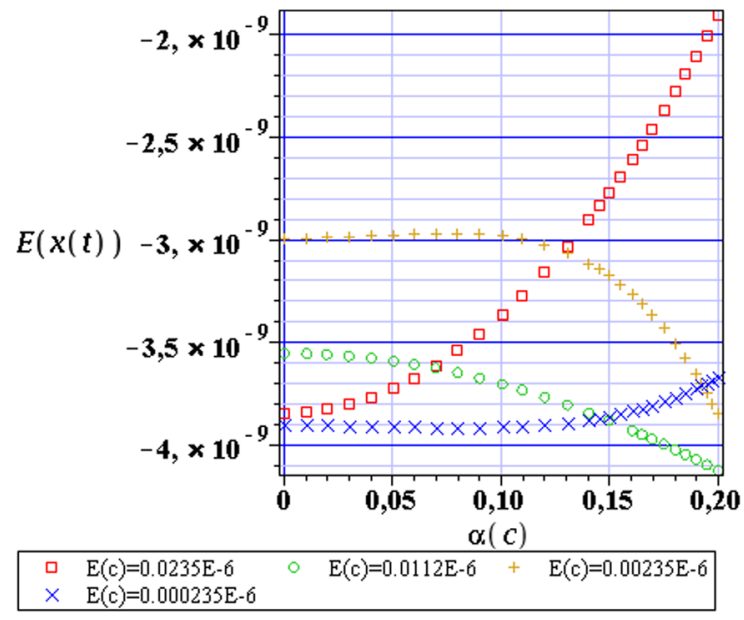

Fig. 9 Expectations of the displacements [m]

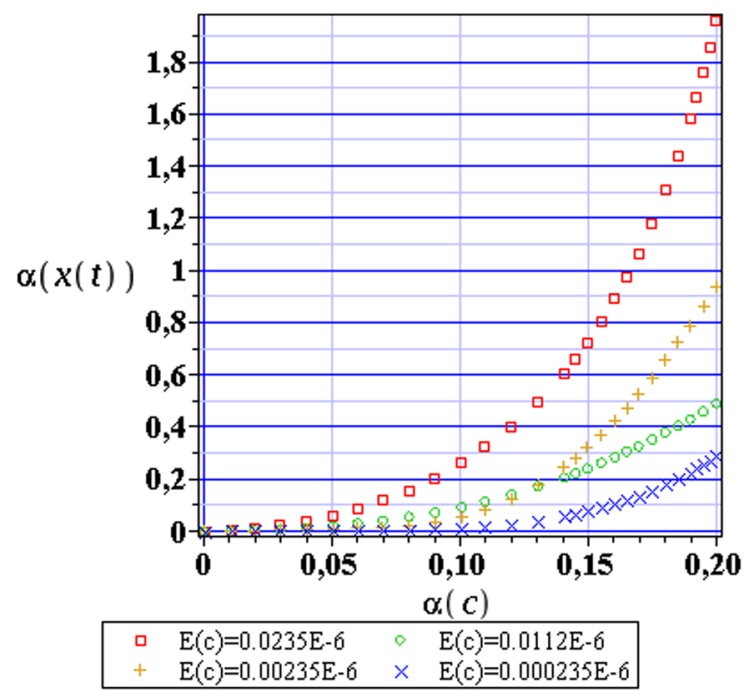

Fig. 10 Coefficients of variation for the displacements

respect to the input coefficient of variation. This expectation seems to be almost insensitive to the input $\mathrm{CoV}$ when reaches its minimum value, $\mathrm{E}[c]=0.000235 \mathrm{E}-6$ and then variations of the resulting $\mathrm{E}[x(t)]$ w.r.t. $\alpha(\mathrm{c})$ systematically increase together with $\mathrm{E}[c]$. Extreme value of the mean damping corresponds to highly nonlinear increase of the expected value $\mathrm{E}[\mathrm{x}(\mathrm{t})]$ that increases almost twice when an uncertainty in c changes from 0.0 up to its extreme for 0.20 . It is interesting that these fluctuations cannot be simply neglected like in classical elasticity theory and elasto-

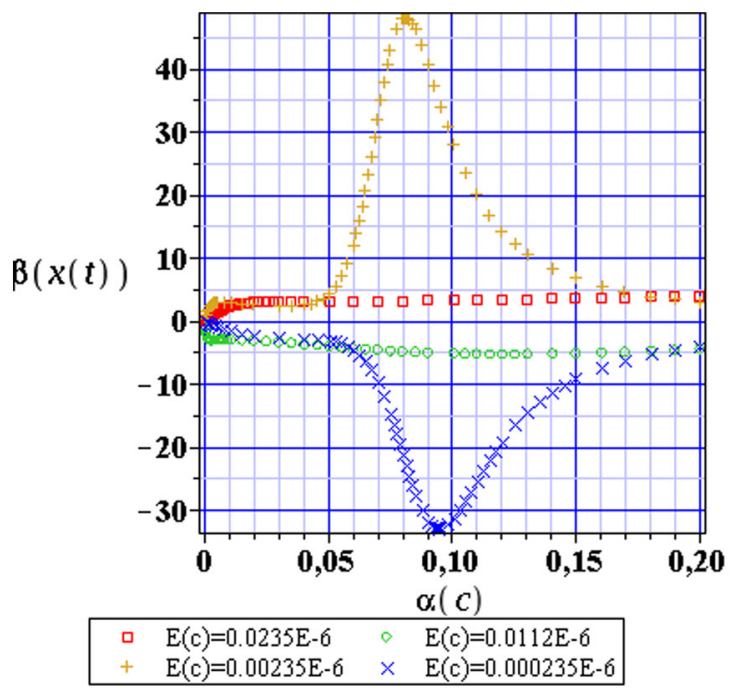

Fig. 11 Skewness of the displacements

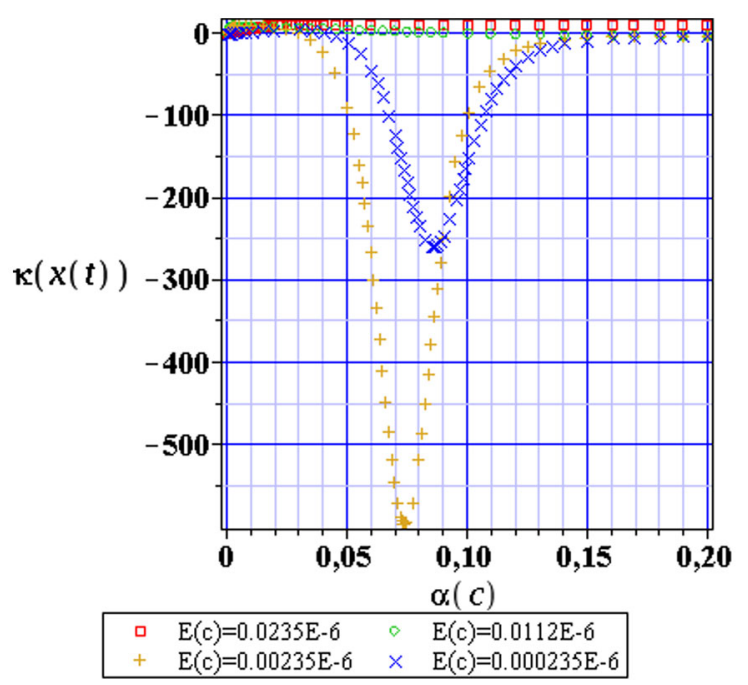

Fig. 12 Kurtosis of the displacements

dynamics with random parameters [10] leading sometimes to an increase or a decrease of the final expectation of $x(t)$. It should be mentioned that the extremely large $\mathrm{E}[\mathrm{c}]$ corresponds to a situation where an absolute value $\mathrm{E}[\mathrm{x}(\mathrm{t})]$ decreases almost twice when $\alpha(\mathrm{c})$ changes its value from 0 adjacent to the deterministic vibration to 0.20 that means the largest possible input deviation in this case. Furthermore, some specific input values lead to an increase and some others - to a decrease of $E(x(t))$ for larger random fluctuations in this parameter $c$; they can increase or decrease almost twice for this specific range of an input $\alpha(c)$. 
Table 1 Approximation errors in the Least Squares Method

\begin{tabular}{lrlll}
\hline Order & Correlation & RMS error & Squares sum & Fitting variance \\
\hline 2 & 0.944648 & $6.50235 \mathrm{E}-12$ & $4.65126 \mathrm{E}-22$ & $4.65228 \mathrm{E}-23$ \\
3 & 0.999390 & $7.22270 \mathrm{E}-13$ & $5.76870 \mathrm{E}-24$ & $5.86760 \mathrm{E}-25$ \\
4 & 0.999571 & $6.09214 \mathrm{E}-13$ & $4.11380 \mathrm{E}-24$ & $2.90649 \mathrm{E}-24$ \\
5 & -0.594698 & $7.08217 \mathrm{E}-10$ & $5.51726 \mathrm{E}-18$ & $2.45674 \mathrm{E}-19$ \\
6 & -0.924625 & $8.02584 \mathrm{E}-9$ & $7.08554 \mathrm{E}-16$ & $4.98402 \mathrm{E}-16$ \\
7 & 0.936636 & $8.11866 \mathrm{E}-7$ & $7.25038 \mathrm{E}-12$ & $3.53456 \mathrm{E}-12$ \\
8 & -0.942430 & $8.61679 \mathrm{E}-5$ & $8.16740 \mathrm{E}-8$ & $1.25495 \mathrm{E}-6$ \\
9 & -0.937340 & $6.38454 \mathrm{E}-4$ & $4.48386 \mathrm{E}-6$ & $6.55977 \mathrm{E}-7$ \\
\hline
\end{tabular}

It is noticeable and quite clear that generally the larger the input mean value of $\mathrm{c}$, the larger the output coefficient of variation. It is additionally ten times larger than the input one for maximum value of $E[c]$, while is almost equal to the input coefficient $\alpha(c)$ for its minimum expectation. Further, one notices that all the curves describing coefficient of variation of random excitation with respect to the input $\mathrm{CoV}$ are convex. We observe that this convexity is not proportional to the input coefficient $\alpha(c)$ - the trends corresponding to the intermediate values of mean damping intersect with each other below $\alpha(c)=0.15$. It means that larger stochastic fluctuations of this MEMS device vibrations may be initially observed with larger average damping until some limit value of $\alpha(c)$ (smaller dispersion of uncertain damping) and then-for the analogous device with smaller expectation of the damping coefficient (with larger dispersion of uncertain damping); it of course may affect the reliability index of this device. It looks that the resulting random dispersion of the MEMS vibrator depends upon a combination of both expectation and coefficient of variation of the input uncertainty in damping unlike in the linear systems with random parameter(s) where it is driven by the input $\mathrm{CoV}$ by only. Higher order statistics given in Figs. 11 and 12, namely skewness and kurtosis, are basically different than these corresponding to the Gaussian distribution. They are additionally really very sensitive with respect to the input coefficient of variation and, surprisingly, exhibit some extreme values for about $\alpha(c)=0.075 \div 0.10$ having the distributions a little bit similar to the bell shaped curve. These extremes correspond to smaller values of expected value of damping, while larger damping correspond to a very stable results for all $\alpha(c) \in[0.00,0.20]$. It means that extreme values of the damping coefficient usually result in the probability distribution of dynamic excitation that looks close to the Gaussian one, while intermediate randomness in parameter $c$ may lead to dramatic increase of both skewness and kurtosis. Let us note also that there are both positive and negative extremes of these coefficients computed for the neighboring values of $\mathrm{E}[c]$.

\section{Concluding remarks}

1. Stochastic perturbation-based numerical solution to the Duffing equation originating from the Taylor expansion of the general order has been proposed in this paper to analyze the vibrations of a micro-resonator with random damping coefficient adopted as Gaussian input parameter. The first four probabilistic moments and coefficients of the displacements and velocities have been determined numerically using the additional implementation in symbolic computing system MAPLE. Numerical solution in the symbolic algebra context provided with the use of the Runge-Kutta-Fehlenberg method has been linked with the non-weighted Least Squares Method, where polynomial stochastic response with respect to the randomized damping has been assumed. A choice of the polynomial order has been made after computation of the RMS error and the correlation associated to the LSM itself. This two-fold minimization enabled to detect that the output expectations of structural displacements are extremely sensitive to the input coefficient of variation. Additionally, the output $\mathrm{CoV}$ may be 
even more than ten times larger than the input one and depends also very much upon the input random parameter expectation. Higher order statistics are rather very distant from these typical for the Gaussian distribution. Consideration of the stochastic damping has deep practical significance and should be extended further towards timedependent uncertainty, i.e. in the form of time series with random coefficients to model the aging process in the MEMS devices.

2. It can be mentioned further that the computational technique proposed is similar to the polynomial chaos approach presented in [1], but instead of lower order polynomials for several random inputs employs a single variable polynomial with higher order terms [10]. Its further development towards multiple randomness sources is a relatively easy task. The essential difference to this method is that we further provide partial differentiation of the system response with respect to the random input and modify classical definition of the probability theory towards the Taylor expansions with random coefficients. It should be mentioned that the computational cost is decisively smaller than for the remaining methods, especially taking into account a significant time consumption in the Monte-Carlo simulations (more than ten times as has been demonstrated here). Our computational strategy may straightforwardly serve for stochastic time-dependent reliability analysis [6] if only the allowable displacements or velocities for some limit function could be defined for this system. Since the method looks promising, it can be further used to make Stochastic Finite Element Method implementations with the existing multiphysics commercial FEM codes. Further applications towards stochastic modeling of the uncertainty adhesion [2] are also possible but they need an implementation of the entire random field approach defined for the adhesive plate and its inclusion into the equations of the model. Stochastic perturbation-based approach may be also of paramount importance in computational modeling of fatigue phenomena in MEMS devices [3], but it needs some prior SFEM realization.

Open Access This article is distributed under the terms of the Creative Commons Attribution License which permits any use, distribution, and reproduction in any medium, provided the original author(s) and the source are credited.

\section{References}

1. Agarwal N, Aluru NR (2009) Stochastic analysis of electrostatic MEMS subjected to parameter variations. J Micoelectromech Syst 18(6):1454-1468

2. Ardito R, Corigliano A, Frangi A (2013) Modelling of spontaneous adhesion phenomena in micro-electro-mechanical systems. Eur J Mech A/Solids 39:144-152

3. Bomidi JAR, Weinzapfel N, Sadeghi F (2012) Three-dimensional modelling of intergranular fatigue failure of fine grain polycrystalline metallic MEMS devices. Fatigue Fract Eng Mater Struct 35(11):1007-1021

4. Ghanem RG, Spanos PD (2003) Stochastic finite elements. Dover Publishers, New York

5. Ghodssi R et al (eds) (2011) MEMS materials and processes handbook. Springer, Berlin

6. Hartzell AL, da Silva MG, Shea HR (2011) MEMS reliability. Springer, Berlin

7. Ilin EA, Kehrbusch J, Radzio B, Oesterschulze E (2011) Analytical model of the temperature dependent properties of microresonators immersed in a fluid. J Appl Phys 109:33519

8. Jalalahmadi B, Sadeghi F, Peroulis D (2009) A numerical fatigue damage model for life scatter of MEMS devices. J Microeletromech Syst 18(5):1016-1031

9. Kaajakari V, Mattila T, Oja A, Seppa H (2004) Nonlinear limits for single-crystal silicon microresonators. IEEE J Microelectromech Syst 13:715-724

10. Kamiński M (2013) The stochastic perturbation method for computational mechanics. Wiley, Chichester

11. Kamiński M, Corigliano A (2012) Sensitivity, probabilistic and stochastic analysis of the thermo-piezoelectric phenomena in solids by the stochastic perturbation technique. Meccanica 47:877-891

12. Kapitaniak T, Bishop S (1999) Dictionary of nonlinear dynamics. Wiley, Chichester

13. Kozyreff G, Dominguez Juarez JL, Martorell J (2008) Whispering-gallery-mode phase matching for surface second-order nonlinear optical processes in spherical microresonators. Phys Rev A 77:043817

14. Landau LD, Lifshitz EM (1999) Mechanics, 3rd edn. Butterworth-Heinemann, Oxford

15. Matsko AB (2009) Practical applications of microresonators in optics and photonics. CRC Press, Boca Raton, Florida

16. Mőller B, Beer M (2004) Fuzzy Randomness. Uncertainty in Civil Engineering and Computational Mechanics. Springer, Berlin

17. Muscolino G (1988) Non-stationary pre-envelope covariances of nonclassicaly damped systems. J Sound Vib 149:107-123

18. Papadrakakis M, Stefanou G, Papadopoulos V (eds) (2011) Computational methods in stochastic mechanics. Springer, New York

19. Piszczek K, Nizioł J (1986) Random vibration of mechanical syastems. Wiley, New York 
20. Settineri D, Falsone A (2014) An APDM-based method for the analysis of systems with uncertainties. Comput Methods Appl Mech Eng 278:828-852

21. Tamazin M, Noureldin A, Korenberg MJ (2013) Robust modeling of low-cost MEMS sensor errors in mobile devices using fast orthogonal search. J Sens. Article ID 101820. http://dx.doi.org/10.1155/2013/101820

22. Tocchio A, Comi C, Langfelder G, Corigliano A, Longoni A (2011) Enhancing the linear range of MEMS resonators for sensing applications. IEEE Sens 11(12):3202-3210
23. Verhoosel CV (2009) Multiscale and probabilistic modelling of micro-electromechanical systems. PhD thesis, TU Delft, Rotterdam

24. de Visser PJ et al (2011) Number fluctuations of sparse quasiparticles in a superconductor. Phys Rev Lett 106:167004. doi:10.1103/PhysRevLett.106.167004

25. Waldner JB (2008) Nanocomputers and swarm intelligence. Wiley, New York 